\title{
EGF-like domains with a C-x-D-x(4)-Y-x-C motif
}

\begin{abstract}
The epidermal growth factor (EGF)-like domain is one of most common domains found in extracellular proteins and many proteins contain multiple EGF-like domains in tandem. Variations in the amino acid sequences of EGF-like domains make their study difficult and the characteristics of EGF-like domains are poorly understood. This mini-review introduces a putative subfamily of EGF-like domains with the consensus amino acid sequence $\mathrm{C}-\mathrm{x}-\mathrm{D}-\mathrm{x}(4)-\mathrm{Y}-\mathrm{x}-\mathrm{C}$. This type of EGF-like domain in Del-1 and vitamin-K dependent coagulation factors has several features in common, such as increasing the efficiency of endocytosis-dependent transfection, attenuating cell adhesion, and inducing apoptosis. This motif is also found in other clinically important proteins such as Notchs, Notch ligands, and low-density lipoprotein receptor. Finally, translational research on this motif is discussed.
\end{abstract}

Volume 2 Issue 2 - 2018

\author{
Chiaki Hidai \\ Department of Biomedical Sciences, Nihon University School of \\ Medicine, Japan
}

\begin{abstract}
Correspondence: Chiaki Hidai, Division of Physiology, Department of Biomedical Sciences, University School of Medicine, 30-I Oyaguchi-kamicho, Itabashi-ku, Tokyo 173-86 I0, Japan, Tel +8I-3-3972-8I I I, Ext 2236, Fax +8I-3-3972-8292, Email hidai.chiaki@nihon-u.ac.jp
\end{abstract}

Received: April 0I, 2018 | Published: April 24, 2018

Keywords: EGF, del-1, vitamin-K dependent coagulation factor, notch, LDLR

Abbreviations: EGF, epidermal growth factor; LDLR, lowdensity lipoprotein receptor; $\mathrm{FH}$, autosomal dominant form of familial hypercholesterolemia; HEGF, human EGF; cEGF, compliment $\mathrm{C} 1 \mathrm{r}$ EGF; cbEGF, calcium binding EGF; ECM, extracellular matrix; ARH, autosomal recessive hypercholesterolemia; PCSK9, Proprotein convertase subtilisin/kexin type 9

\section{Introduction}

The epidermal growth factor (EGF)-like domain was first identified in pro-epidermal growth factor in 1983 and since then many proteins with EGF-like domains have been reported to be involved in pathogenesis. ${ }^{1,2}$ Pro-EGF is significantly related with oncogenesis. ${ }^{3,4}$ Mutations of EGF-like domains in fibrillin-1, low-density lipoprotein receptor (LDLR), and coagulation factor IX (FIX) are the causes of Marfan syndrome, the autosomal dominant form of familial hypercholesterolemia (FH), and hemophilia B, respectively. ${ }^{5-8}$ EGFlike domains are found in numerous secreted extracellular proteins, except for the endoplasmic transmembrane protein prostaglandin $\mathrm{G} / \mathrm{H}$ synthase. ${ }^{9}$ Extracellular proteins are easily accessed by drugs and thus are good therapeutic targets, making studies of EGF-like domains medically significant.

Currently, 2,662 EGF-like domains are identified in about 640 proteins in the SwissPro database. ${ }^{10}$ Most of these proteins are from animals but some are from plants and slim molds. Several proteins contain multiple EGF-like domains in tandem. For example, fibrillin-1 has 47 and Notch-1 has 36 EGF-like domains. EGF-like domains comprise a rich variety of amino acid sequences between conserved cysteine residues. The number and variation in EGF-like domain sequences complicates understanding the overall character of EGF-like domains. ${ }^{11}$ Dividing EGF-like domains into subfamilies would aid investigation of their functions.

This short manuscript reviews reports regarding a putative novel subfamily of EGF-like domains containing the amino acid sequence C-x-D-x(4)-Y-x-C (referred to as the DXXXXY motif in this review) and describes the characteristics of this motif. First, we describe the general properties of EGF-like domains, and then we review reports regarding the DXXXXY motif using information from the UniPro database. The DXXXXY motif shows a variety of functions in vitro and in vivo, and is found in several medically important proteins such as coagulation factors, low density lipoprotein receptor, and Notch related proteins. We conclude by discussing the significance of the DXXXXY motif in these proteins.

\section{EGF-like domain}

The EGF-like domain typically consists of 30-45 amino acids, including six cysteine residues that form three disulfide bonds. The domain has two $\beta$-sheets, with the major sheet at the $\mathrm{N}$-terminus and the minor sheet at the $\mathrm{C}$-terminus. The three disulfides are in the major sheet. In addition to the basic structure, distinct domain subtypes and post transcriptional modifications have been identified.

\section{Human EGF-like (hEGF) domain and compliment CIr (cEGF) domain}

The two main types of EGF-like domains are the human EGF-like (hEGF) domain and the compliment C1r EGF-like (cEGF) domain. Wouters et al. discussed the differences between these two groups with regards to their amino acid sequences and biological characteristics based on their experimental data and database research. ${ }^{10}$ The two domains have clear differences in tertiary structure. ${ }^{12}$ Additionally, the 6 th cysteine in $\mathrm{hEGF}$ is located in the $\beta$-turn of the minor sheet whereas the 6th cysteine in cEGF is located on the second strand of the sheet itself. There are 8-9 amino acids between the 5th and $6^{\text {th }}$ cysteines in hEGF but more than 10 amino acids between these cysteines in cEGF. Some proteins contain only hEGFs (e.g., Notchs), some proteins contain only cEGFs (e.g., LDLR), and some proteins contain both (e.g., fibrillins). The different functions of these two subtypes are poorly understood.

\section{Calcium binding}

The calcium binding EGF-like domain (cbEGF) consists of 45 amino acids and its consensus sequence is [DEQN]-x-[DEQN](2)$\mathrm{C}-\mathrm{x}(3,14)-\mathrm{C}-\mathrm{x}(3,7)-\mathrm{C}-\mathrm{x}-[\mathrm{DN}]-\mathrm{x}(4)-[\mathrm{FY}]-\mathrm{x}-\mathrm{C} .{ }^{13}$ The affinity of this sequence for calcium is independent of differences between hEGF and cEGF. Calcium binding to cbEGF is critical for the functions of proteins. For example, fibrillin-1 contains 47 EGF domains, of 
which 43 belong to cbEGF, and mutations affecting calcium binding cause Marfan syndrome. ${ }^{7}$ These mutations increase susceptibility to proteolysis and thus calcium binding may help maintain the native conformations of these proteins. ${ }^{14}$

\section{O-glycosylation}

O-glycosylation modifications of amino acid residues have been reported in hEGFs, including Notch, and the functions of these modifications have been well studied in Notch EGF-like domains. ${ }^{15-17}$ Typically, threonine and serine just before the 4th cysteine in EGFlike domains are modified with $\mathrm{O}$-fucose and the second serines after the 1st cysteine are modified with O-glucose. O-linked glycosylation by GALNT-11 is involved in determining left/right symmetry: glycosylation promotes the activation of Notch-1, possibly by promoting cleavage by ADAM17, modulating the balance between motile and immotile (sensory) cilia at the left-right organizer (LRO). ${ }^{14}$ O-glycosylations on EGF-like domains 11, 12 and 13 contain both O-linked fucose and O-linked glucose which interact with residues in DLL4. ${ }^{18}$

\section{$\beta$-hydroxylation}

Post-translational hydroxylation of aspartic acid or asparagine to form erythro- $\beta$-hydroxyaspartic acid or erythro- $\beta$-hydroxyasparagine has been identified in several EGF-like domains. The consensus amino acid sequence for $\beta$-hydroxylation is $\mathrm{C}-\mathrm{x}-[\mathrm{DN}]-\mathrm{x}(4)-[\mathrm{FY}]-\mathrm{x}-\mathrm{C} .{ }^{19}$ There is a one-to-one correspondence between the hydroxylation of aspartic acid and hEGF, and of the hydroxylation of asparagine and cEGF. ${ }^{10}$ The significance of $\beta$-hydroxylation is poorly understood. Aspartic acid residues in the DXXXXY motifs of Notch are $\beta$-hydroxylated by aspartyl $\beta$-hydroxylase. Gene targeting of aspartyl $\beta$-hydroxylase for $\beta$-hydroxylation results in cleft palate and the fusion of digits. Additionally, in the presence of APC (adenomatous polyposis coli) mutations, gene targeting of aspartyl $\beta$-hydroxylase increases the number of polyps in intestines. The $\beta$-hydroxylation of Notch EGFlike domains might suppress tumors. ${ }^{20}$

\section{DXXXXY motif}

The consensus amino acid sequence for $\beta$-hydroxylation of EGF-like domains, C-x-[DN]-x(4)-[FY]-x-C, indicates four ways, DXXXXF, DXXXXY, NXXXXF, and NXXXXY. One sequence, C-X-D-x(4)-Y-x-C, may form a putative subfamily. The EGF-like domains from Del-1 and FIX increase the gene transfer efficiency of non-viral vectors via endocytosis in vitro and in vivo. ${ }^{21}$ The EGFlike domains in Del-1 and FIX are hEGF and cbEGF types with the DXXXXY motif. The mutation of aspartic acid to asparagine or tyrosine to phenylalanine decreases the activities of Del-1. Other proteins containing the DXXXXY motif are discussed below.

\section{Del-I}

Del-1 is an extracellular matrix (ECM) protein expressed by embryonic endothelial cells. ${ }^{22}$ Over-expression of Del-1 enhances the remodeling of blood vessels in vasculogenesis. ${ }^{23}$ In adults, Del1 regulates leucocyte adhesion to pulmonary endothelial cells in inflamed tissues. ${ }^{24}$ Del-1 contains three EGF-like domains in the $\mathrm{N}$-terminus and two discoidin domains in the C-terminus. The EGFlike domains in Del-1 are all hEGF. The second EGF-like domain contains an RGD integrin-binding motif and the third EGF-like domain has a DXXXXY motif. Discoidin domains have been reported to bind to anionic phospholipids on the surface of cells. ${ }^{25}$

A recombinant version of the 3rd EGF-like domain of Del-1 induces endocytosis, pinocytosis, and phagocytosis, attenuates cell adhesion to ECM, and induces caspase-dependent apoptosis in vitro and in vivo. ${ }^{26-28}$ The EGF-like domain with a DXXXXY motif serves as a trans-element for these functions. Del-1 accumulates in ECM, and a domain for adhesion to ECM has been localized to the 1st discoidin domain..$^{29}$ The adhesion activity of the 1st discoidin domain is significantly enhanced by the adjacent 3rd EGF-like domain. The EGF-like domain with a DXXXXY motif serves as a cis-element for this function. Based on the finding that the 3rd EGF-like domain and the 1st discoidin domain accumulate in ECM, induce apoptosis, and improve transfection efficiency, Kitano et al. treated tumor explant model mice with cDNA of the 3rd EGF-like domain and the 1st discoidin domain using a non-viral vector. ${ }^{30,31}$ The transgene product accumulated in ECM and induced apoptosis in tumors, indicating that such gene therapy can significantly suppresses tumor growth and improve life prognosis.

\section{Coagulation factors VII, IX, and X}

Coagulation factors VII (FVII), IX (FIX) and X (FX) are highly homologous vitamin-K dependent enzymes that are essential for coagulation. ${ }^{8,32}$ They have a $\gamma$-carboxyglutamic acid (Gla) domain at the $\mathrm{N}$-terminus that is essential for binding to phosphatidylserine in the cell membrane. Two EGF-like domains are adjacent to the Gla domain. The 1st EGF-like domain is a hEGF and cbEGF domain with the DXXXXY motif and the 2nd is a cEGF but not a cbEGF domain..$^{13}$ A peptidase domain that cleaves other coagulation factors is adjacent to the 2nd EGF-like domain. Mutation of FIX causes hemophilia B and mutation of the 1st EGF-like domain disrupts calcium binding and may cause hemophilia $\mathrm{B} .{ }^{33}$ In the absence of calcium, the 1st EGF-like domain in FIX adopts a confirmation that locks the Gla domain in a fixed position, restricting movement of the Gla and cbEGF domains. ${ }^{34-36}$ Calcium binding to cbEGF induces a conformational change that may change protein function, such as enzymatic activity and binding to other molecules. For this function, the EGF-like domain with a DXXXXY motif serves as a cis-element.

A recombinant version of the 1st EGF-like domain of FIX suppresses cell adhesion to ECM, increases cell migration, and activates caspase-3..$^{37}$ The 1st EGF-like domains of FVII and FX share these functions. The EGF-like domain with a DXXXXY motif serves as a trans-element for these functions. Additionally, the recombinant 1st EGF-like domain of FIX induces the exposure of phosphatidylserine and the clustering of lipid rafts in the cell membrane that likely contribute to endocytosis. ${ }^{38}$ Because coagulation factors are generally activated in wounds, these functions of the 1st EGF-like domains of coagulation factors may contribute to wound healing and immunological reactions.

There are three other vitamin-K dependent coagulation-related proteins: protein $\mathrm{C}$, protein $\mathrm{S}$, and protein $\mathrm{Z} .{ }^{32}$ They also consist of a Gla domain, an EGF-like domain, and a peptidase domain, similar to FVII, FIX and FX. However, they negatively regulate coagulation. Interestingly, protein $\mathrm{C}$ and protein $\mathrm{S}$ have an NXXXXY sequence instead of DXXXXY. Protein $\mathrm{Z}$ has a DXXXXY motif but is not a cbEGF.

\section{Other proteins with the $D X X X X Y$ motif}


Other than Del-1 and vitamin-K dependent coagulation factors, there are several other proteins with the DXXXXY motif in EGF-like domains (Table 1). There are 31 proteins with the DXXXXY motif in the UniPro database and they can be categorized into 5 groups: ECM-associated proteins, vitamin-K dependent coagulation factors, Notch-related proteins, Lipoprotein transport-related proteins, and other membrane proteins. All contain between one to $12 \mathrm{DXXXXY}$ motifs. Their associated domains are often involved in binding other molecules. The four amino acids residues between the aspartic acid and tyrosine in DXXXXY share several characteristics (Table 2). Many of the residues are aliphatic, non-polar, neutral amino acids and thus the DXXXXY motif should be hydrophobic. ${ }^{39}$

Table I List of proteins with the DXXXXY motif in ECG-like domains

\begin{tabular}{|c|c|c|c|c|}
\hline$\#$ & UniProtKB \# & ECM associated proteins & No of DXXXXY motifs & $\begin{array}{l}\text { Associated } \\
\text { domains }\end{array}$ \\
\hline 1 & O43854 & Dell & 1 & Discoidin \\
\hline 2 & 075093 & Slit homolog I protein & 1 & \\
\hline 3 & 094813 & Slit homolog 2 protein & 1 & Laminin G, LRR \\
\hline 4 & 075094 & Slit homolog 3 protein & 1 & Laminin G, LRR \\
\hline 5 & P35556 & Fibrillin-2 & 1 & TB \\
\hline 6 & Q75N90 & Fibrillin-3 & 1 & $\mathrm{~TB}$ \\
\hline 7 & Q8TER0 & $\begin{array}{l}\text { Sushi, nidogen and EGF-like domain- } \\
\text { containing protein }\end{array}$ & 3 & Sushi, Follistatin \\
\hline 8 & Q4LDE5 & $\begin{array}{l}\text { Sushi, von Willebrand factor type A, EGF and } \\
\text { pentraxin domain-containing protein I }\end{array}$ & 2 & Sushi \\
\hline \multicolumn{5}{|c|}{ Vitamin $\mathrm{K}$ dependent coagulation factors } \\
\hline 9 & P08709 & Coagulation factorVII & 1 & Gla \\
\hline 10 & P00742 & Coagulation factor $X$ & 1 & Gla \\
\hline 11 & P00740 & Coagulation factor IX & 1 & Gla \\
\hline 12 & P22891 & Vitamin K-dependent protein Z & 1 & Gla \\
\hline \multicolumn{5}{|c|}{ Notch related proteins } \\
\hline 13 & P4653I & Notch I & 12 & Ank \\
\hline 14 & Q0472I & Notch 2 & 10 & Ank \\
\hline 15 & Q9UM47 & Notch 3 & 3 & Ank \\
\hline 16 & Q99466 & Notch 4 & 2 & Ank \\
\hline 17 & Q00548 & Delta-like protein I & 1 & DSL \\
\hline 18 & Q9NYJ7 & Delta-like protein 3 & 1 & DSL \\
\hline 19 & Q9NR61 & Delta-like protein 4 & 1 & DSL \\
\hline 20 & P78504 & Protein Jagged-I & 3 & DSL \\
\hline 21 & Q9Y219 & Protein Jagged-2 & 1 & DSL \\
\hline 22 & Q8NFT8 & $\begin{array}{l}\text { Delta and Notch-like epidermal growth factor } \\
\text { related receptor }\end{array}$ & 2 & Follistatin \\
\hline \multicolumn{5}{|c|}{ Lipoprotein transport related proteins } \\
\hline 23 & P0II30 & Low-density lipoprotein receptor & 1 & LDLR-I \\
\hline 24 & P98I55 & Very low-density lipoprotein receptor & 1 & LDLR-I \\
\hline 25 & Q9NZR2 & $\begin{array}{l}\text { Low-density lipoprotein receptor -related } \\
\text { protein IB }\end{array}$ & 1 & LDLR-I \\
\hline 26 & O60494 & Cubilin & 1 & CUB \\
\hline \multicolumn{5}{|c|}{ Other transmembrane proteins } \\
\hline 27 & P82279 & Protein crumbs homolog I & 4 & Laminin G \\
\hline 28 & Q5IJ48 & Protein crumbs homolog 2 & 5 & Laminin G \\
\hline 29 & Q9ULBI & Neurexin I-alpha & 1 & Laminin G \\
\hline 30 & Q9NYQ6 & $\begin{array}{l}\text { Cadherin EGF LAG seven-pass G-type } \\
\text { receptor I }\end{array}$ & 1 & Laminin G \\
\hline 31 & Q727M0 & $\begin{array}{l}\text { Multiple epidermal growth factor-like domains } \\
\text { protein } 8\end{array}$ & 1 & CUB, PSI \\
\hline
\end{tabular}

Table 2 Frequency of amino acids residues at the four positions between $\mathrm{D}$ and $\mathrm{Y}$ 


\begin{tabular}{llllllll}
\hline $\mathbf{I}^{\text {st }}$ & & $\mathbf{2}^{\text {nd }}$ & & $\mathbf{3}^{\text {rd }}$ & \multicolumn{3}{c}{$\mathbf{4}^{\text {th }}$} \\
\hline AA & $\%$ & AA & $\%$ & AA & $\%$ & AA & $\%$ \\
\hline G & 23 & V & 33 & N & 40 & G & 47 \\
L & 20 & I & 17 & G & $\%$ & S & 27 \\
Q & 20 & P & 10 & A & 8 & T & 8 \\
& & L & 10 & D & 8 & &
\end{tabular}

The three most frequent amino acids are presented.AA, amino acid.

Notch-1 contains 36 EGF-like domains. Human and Xenopus Notch-1 have 12 DXXXXY motifs and mouse, zebrafish, and drosophila Notch-1 have 11 DXXXXY motifs mainly in a middle section of their extracellular domain. All EGF-like domains with the DXXXXY motif are hEGF, and 11 of 12 DXXXXY motifs are cbEGF in human Notch-1. Several proteins that are Notch ligands also have DXXXXY motifs and both Notchs, and their ligand proteins play critical roles in development, cancer, and angiogenesis. It is well documented that EGF-like domains 11-12 are necessary and sufficient for ligand binding. ${ }^{15,18,40}$ Additionally, a study in Drosophila suggests that EGF-like domains 24-29 are important in ligand-dependent receptor activation. ${ }^{41}$ However, the roles of the DXXXXY motifs in Notch-related proteins are poorly understood.

LDLR is essential for the uptake of LDL by hepatocytes via endocytosis. Mutations of LDLR cause the autosomal dominant form of familial hypercholesterolemia $(\mathrm{FH})$ and autosomal recessive hypercholesterolemia (ARH). ${ }^{5,42}$ LDLR has an N-terminus LDL binding domain followed by three EGF-like domains that are all cEGF. The 1st EGF-like domain has the amino acid sequence GTNECLDNNGGCSHVCNDLKIGYECLC, an atypical cbEGF sequence, and contains a DXXXXY motif. The 1st EGF-like domain of LDLR plays an important role in pathogenesis of FH. Wild type LDLR is recycled and spared from degradation after endocytosis. ${ }^{43}$ A mutant LDLR protein lacking the EGF-like domains is degraded in lysosomes after endocytosis, thereby resulting in a decrease in the levels of LDLR. The decrease of LDLR decreases the uptake of LDL by hepatocytes and increases the LDL concentration in serum. Proprotein convertase subtilisin/kexin type 9 (PCSK9) was originally identified as a protein that induces neuronal apoptosis and then was recognized as a key molecule in the regulation of serum LDL levels. ${ }^{44,45}$ The binding of PCSK9 to the 1st EGF-like domain of LDLR increases the degradation of LDLR. ${ }^{46}$ The 1st EGF-like domain of LDLR serves as a switch of endosome with LDLR between degradation or recycle. Blocking antibody to PCSK9 is clinically used as a drug to treat $\mathrm{FH} .{ }^{47}$

\section{Conclusion}

EGF-like domains with the DXXXXY motif in Del-1 and vitamin-K dependent coagulation factors share common functions. The DXXXXY motif may allow categorization into a subfamily containing the $\beta$-hydroxylation motif $\mathrm{C}-\mathrm{x}-[\mathrm{DN}]-\mathrm{x}(4)-[\mathrm{FY}]-\mathrm{x}-\mathrm{C}$. Testing this hypothesis requires empirical examination of the functions of EGF-like domains with the DXXXXY motif in other proteins. The EGF-like domains in FIX and Del-1 have been found to be cis-acting elements that regulate the shapes and functions of adjacent domains. Additionally, they are trans-acting. The binding targets of EGF-like domains require further investigation. If the 1st EGF of LDLR has the same functions as the DXXXXY motif, then PCSK9 would be a binding partner of EGF-like domains. Trans-acting elements could have a clinical advantage from the perspective of administration routes. The EGF-like domain of Del-1 enables in vivo gene transfer into brain using a non-viral vector via endocytosis and efficiently improved the life prognosis of a mouse implant tumor model via apoptosis. In addition to their use as therapeutic reagents, EGF-like domains with the DXXXY motif are interesting as pathogenetic elements because Del-1, coagulation factors, Notch-related proteins and lipoprotein transport-related proteins are linked to the vascular system and diseases. ${ }^{22-24,48,49}$ Notch 1 and coagulation factors have been reported to exert stimulatory influences on atherosclerosis. PCSK serves as an atherosclerotic factor via binding to DXXXXY motif of LDLR. Therefore DXXXXY motif could play an integral role in the pathogenesis of atherosclerosis. Further studies of the potential clinical utility of DXXXXY motif functions are required.

\section{Acknowledgements}

None.

\section{Conflict of interest}

The authors declare no conflict of interest.

\section{References}

1. Gray A, Dull TJ, Ullrich A. Nucleotide sequence of epidermal growth factor cDNA predicts a 128,000-molecular weight protein precursor. Nature. 1983;303(5919):722-725.

2. Scott J, Urdea M, Quiroga M, et al. Structure of a mouse submaxillary messenger RNA encoding epidermal growth factor and seven related proteins. Science. 1983;221(4607):236-240

3. Esparis-Ogando A, Montero JC, Arribas J, et al. Targeting the EGF/HER Ligand-Receptor System in Cancer. Curr Pharm Des. 2016;22(39):5887-5898.

4. Lindsey S, Langhans SA. Epidermal growth factor signaling in transformed cells. Int Rev Cell Mol Biol. 2015;314:1-41.

5. Abifadel M, Rabes JP, Jambart S, et al. The molecular basis of familial hypercholesterolemia in Lebanon: spectrum of LDLR mutations and role of PCSK9 as a modifier gene. Hum Mutat. 2009;30(7):E682691.

6. Hobbs HH, Brown MS, Goldstein JL. Molecular genetics of the LDL receptor gene in familial hypercholesterolemia. Hum Mutat. 1992;1(6):445-466.

7. Zeyer KA, Reinhardt DP. Engineered mutations in fibrillin-1 leading to Marfan syndrome act at the protein, cellular and organismal levels. Mutat Res Rev Mutat Res. 2015;765:7-18.

8. Lillicrap D. FIX It in One Go: Enhanced Factor IX Gene Therapy for Hemophilia B. Cell. 2017;171(7):1478-1480.

9. Otto JC, Dewitt DL, Smith WL. N-glycosylation of prostaglandin endoperoxide synthases- 1 and -2 and their orientations in the endoplasmic reticulum. $J$ Biol Chem. 1993;268(24):18234-18242.

10. Wouters MA, Rigoutsos I, Chu CK, et al. Evolution of distinct EGF domains with specific functions. Protein Sci. 2005;14(4):1091-1103.

11. Rand MD, Lindblom A, Carlson J, et al. Calcium binding to tandem repeats of EGF- like modules. Expression and characterization of the EGF-like modules of human Notch-1 implicated in receptor-ligand interactions. Protein Sci. 1997;6(10):2059- 2071.

12. Bersch B, Hernandez JF, Marion D, et al. Solution structure of the epidermal growth factor (EGF)-like module of human complement protease $\mathrm{C} 1 \mathrm{r}$, an atypical member of the EGF family. Biochemistry. 1998;37(5):1204-1214.

13. Handford PA, Baron M, Mayhew M, et al. The first EGF-like domain from human factor IX contains a high-affinity calcium binding site. 
EMBO J. 1990;9(2):475-480.

14. Boskovski MT, Yuan S, Pedersen NB, et al. The heterotaxy gene GALNT11 glycosylates Notch to orchestrate cilia type and laterality. Nature. 2013;504(7480):456-459.

15. Rana NA, Nita-Lazar A, Takeuchi H, et al. O-glucose trisaccharide is present at high but variable stoichiometry at multiple sites on mouse Notch1. J Biol Chem. 2011;286(36):31623-31637.

16. Kakuda S, Haltiwanger RS. Deciphering the Fringe-Mediated Notch Code: Identification of Activating and Inhibiting Sites Allowing Discrimination between Ligands. Dev Cell. 2017;40(2):193-201.

17. Haltom AR, Jafar-Nejad H. The multiple roles of epidermal growth factor repeat O-glycans in animal development. Glycobiology. 2015;25(10):1027-1042.

18. Luca VC, Jude KM, Pierce NW, et al. Structural biology. Structural basis for Notch1 engagement of Delta- like 4. Science. 2015;347(6224):847-853.

19. Rees DJ, Jones IM, Handford PA, et al. The role of betahydroxyaspartate and adjacent carboxylate residues in the first EGF domain of human factor IX. EMBO J. 1988;7(7):2053-2061.

20. Dinchuk JE, Focht RJ, Kelley JA, et al. Absence of post-translational aspartyl beta-hydroxylation of epidermal growth factor domains in mice leads to developmental defects and an increased incidence of intestinal neoplasia. J Biol Chem. 2002;277(15):12970-129777.

21. Kitano H, Hidai C, Kawana M, et al. An epidermal growth factorlike repeat of Dell protein increases the efficiency of gene transfer in vitro. Mol Biotechnol. 2008;39(3):179-185.

22. Hidai C, Zupancic T, Penta K, et al. Cloning and characterization of developmental endothelial locus-1: an embryonic endothelial cell protein that binds the alphavbeta3 integrin receptor. Genes Dev. 1998;12(1):21-33.

23. Hidai C, Kawana M, Habu K, et al. Overexpression of the Dell gene causes dendritic branching in the mouse mesentery. Anat Rec A Discov Mol Cell Evol Biol. 2005;287(2):1165-1175.

24. Choi EY, Chavakis E, Czabanka MA, et al. Del-1, an endogenous leukocyte-endothelial adhesion inhibitor, limits inflammatory cell recruitment. Science. 2008;322(5904):1101-1104.

25. Hanayama R, Tanaka M, Miwa K, et al. Expression of developmental endothelial locus- 1 in a subset of macrophages for engulfment of apoptotic cells. J Immunol. 2004;172(6):3876-3882.

26. Hidai C. Endocytosis: A Structural Components, Functions and Pathways. Nova Biomedical Books, 2010.

27. Kitano H, Kokubun S, Hidai C. The extracellular matrix protein Dell induces apoptosis via its epidermal growth factor motif. Biochem Biophys Res Commun. 2010;393(4):757-761.

28. Kitano H, Mamiya A, Tomomi I, et al. Coagulation factor IX regulates cell migration and adhesion in vitro. Cell Biol Int. 2015;39(10):11621172 .

29. Hidai C, Kitano H, Kokubun S. The Del1 deposition domain can immobilize 3alpha-hydroxysteroid dehydrogenase in the extracellular matrix without interfering with enzymatic activity. Bioprocess Biosyst Eng. 2009;32(5):569-573.

30. Kitano H, Mamiya A, Kokubun S, et al. Efficient nonviral gene therapy with FasL and Dell fragments in mice. $J$ Gene Med. 2012;14(11):642-650.

31. Kitano H, Atsushi Mamiya, Hidai C. Improvement of FasL gene therapy in vitro by fusing the FasL to Dell protein domains. Targets in Gene Therapy. 2011;147-158.

32. Lichtman MA, E Beutler, TJ Kipps, et al. Williams Hematology. 7th ed. McGrawHill: New York, USA, 2006;30(3):1856

33. Stenflo J, Stenberg Y, Muranyi A. Calcium-binding EGF-like modules in coagulation proteinases: function of the calcium ion in module interactions. Biochim Biophys Acta. 2000;1477(1-2):51-63.

34. Vadivel K, Bajaj SP. Structural biology of factor VIIa/tissue factor initiated coagulation. Front Biosci (Landmark Ed). 2012;17:24762494.

35. Sunnerhagen M, Olah GA, Stenflo J, et al. The relative orientation of Gla and EGF domains in coagulation factor $\mathrm{X}$ is altered by $\mathrm{Ca} 2+$ binding to the first EGF domain. A combined NMR-small angle Xray scattering study. Biochemistry. 1996;35(36):11547-11559.

36. Sunnerhagen M, Forsen S, Hoffren AM, et al. Structure of the $\mathrm{Ca}\left(2^{+}\right)$-free Gla domain sheds light on membrane binding of blood coagulation proteins. Nat Struct Biol. 1995;2(6):504-509.

37. Ishikawa T, Kitano H, Mamiya A, et al. The first EGF domain of coagulation factor IX attenuates cell adhesion and induces apoptosis. Biosci Rep. 2016;36(3).

38. Hidai C, Fujiwara Y, Kokubun S, et al. EGF domain of coagulation factor IX is conducive to exposure of phosphatidylserine. Cell Biol Int. 2017;41(4):374-383.

39. Kyte J, Doolittle RF. A simple method for displaying the hydropathic character of a protein. J Mol Biol. 1982;157(1):105-132.

40. Luca VC, Kim BC, Ge C, et al. Notch-Jagged complex structure implicates a catch bond in tuning ligand sensitivity. Science. 2017;355(6331):1320-1324.

41. Sharma A, Rangarajan A, Dighe RR. Antibodies against the extracellular domain of human Notch1 receptor reveal the critical role of epidermal-growth-factor-like repeats $25-26$ in ligand binding and receptor activation. Biochem J. 2013;449(2):519-530.

42. Dvir H, Shah M, Girardi E, et al. Atomic structure of the autosomal recessive hypercholesterolemia phosphotyrosine-binding domain in complex with the LDL-receptor tail. Proc Natl Acad Sci USA. 2012;109(18):6916-6921.

43. Van Der Westhuyzen DR, Stein ML, Henderson HE, et al. Deletion of two growth- factor repeats from the low-density- lipoprotein receptor accelerates its degradation. Biochem J. 1991;277(Pt 3):677682.

44. Lagace TA, Curtis DE, Garuti R, et al. Secreted PCSK9 decreases the number of LDL receptors in hepatocytes and in livers of parabiotic mice. J Clin Invest. 2006;116(11):2995-3005

45. Bingham B, Shen R, Kotnis S, et al. Proapoptotic effects of NARC 1 (=PCSK9), the gene encoding a novel serine proteinase. Cytometry A. 2006;69(11):1123-1131.

46. Kwon HJ, Lagace TA, Mcnutt MC, et al. Molecular basis for LDL receptor recognition by PCSK9. Proc Natl Acad Sci USA. 2008;105(6):1820-1825.

47. Wicinski M, Zak J, Malinowski B, et al. PCSK9 signaling pathways and their potential importance in clinical practice. EPMA $J$. 2017;8(4):391-402.

48. Aquila G, Pannella M, Morelli MB, et al. The role of Notch pathway in cardiovascular diseases. Glob Cardiol Sci Pract. 2013;2013(4):364371.

49. Solanki A, Bhatt LK, Johnston TP. Evolving targets for the treatment of atherosclerosis. Pharmacol Ther. 2018;S0163-7258(18):3002130024 